\title{
Impact of a Post-Discharge Integrated Disease Management Program on COPD Hospital Readmissions
}

\author{
Ashlee N Russo MD, Gayathri Sathiyamoorthy MD, Chris Lau MD, Didem Saygin MD, \\ Xiaozhen Han MSc, Xiao-Feng Wang PhD, Richard Rice RRT, Loutfi S Aboussouan MD, \\ James K Stoller MD MSc FAARC, and Umur Hatipoğlu MD
}

\begin{abstract}
BACKGROUND: Readmission following a hospitalization for COPD is associated with significant health-care expenditure. METHODS: A multicomponent COPD post-discharge integrated disease management program was implemented at the Cleveland Clinic to improve the care of patients with COPD and reduce readmissions. This retrospective study reports our experience with the program. Groups of subjects who were exposed to different components of the program were compared regarding their readmission rates. Multivariate logistic regression analysis was performed to build predictive models for 30- and 90-d readmission. RESULTS: One hundred sixty subjects completed a 90-d follow-up, of which, 67 attended the exacerbation clinic, 16 subjects received care coordination, 51 subjects completed both, and 26 subjects did not participate in any component despite referral. Thirty- and 90-d readmission rates for the entire group were 18.1 and $46.2 \%$, respectively. Thirty- and 90-d readmission rates for the individual groups were: exacerbation clinic, 11.9 and $35.8 \%$; care coordination, 25.0 and $50.0 \%$; both, 19.6 and $41.2 \%$; and neither, 26.9 and $80.8 \%$, respectively. The model with the best predictive ability for 30-d readmission risk included the number of hospitalizations within the previous year and use of noninvasive ventilation ( $C$ statistic of 0.84 ). The model for 90-d readmission risk included receiving any component of the postdischarge integrated disease management program, the number of hospitalizations, and primary care physician visits within the previous year (C statistic of 0.87 ). CONCLUSIONS: Receiving any component of a post-discharge integrated disease management program was associated with reduced 90-d readmission rate. Previous health-care utilization and lung function impairment were strong predictors of readmission. Key words: COPD; integrated disease management; care coordination; readmissions; post-discharge clinic. [Respir Care 2017;62(11):1396-1402. (C) 2017 Daedalus Enterprises]
\end{abstract}

\section{Introduction}

Readmission of patients with COPD remains a vexing problem, with great morbidity for patients and challenges for health-care organizations in the context of bundled

Dr Russo, Dr Sathiyamoorthy, Dr Lau, Dr Saygin, Mr Rice, Dr Aboussouan, Dr Stoller, and Dr Hatipoğlu are affiliated with the Respiratory Institute, Department of Pulmonary Medicine; Ms Han and Dr Wang are affiliated with the Department of Quantitative Health Sciences; and Dr Stoller is affiliated with the Education Institute, Cleveland Clinic, Cleveland, Ohio.

Supplementary material related to this paper is available at http:// www.rcjournal.com.

The authors have disclosed no conflicts of interest. payment. COPD-related costs in the United States have been estimated at approximately $\$ 73$ billion $/ y,{ }^{1}$ with most expenditures due to hospital care. ${ }^{2}$ COPD is also the third leading disease condition associated with hospital readmissions within $30 \mathrm{~d}$ of hospital discharge.$^{3}$ Consequently, the Centers for Medicare and Medicaid Services have tar-

\footnotetext{
Dr Russo presented a version of this work at the American Thoracic Society 2016 International Conference, held May 13-18, 2016, in San Francisco, California.

Correspondence: Umur Hatipoğlu MD, Respiratory Institute, Cleveland Clinic, Mail code A-90, 9500 Euclid Avenue, Cleveland, OH 44195. E-mail: hatipou@ccf.org.
}

DOI: $10.4187 /$ respcare.05547 
geted COPD as a disease for which payment is withheld for readmission within $30 \mathrm{~d}$.

Taken together, integrated disease management interventions improve quality of life and functional capacity and reduce hospital admissions for patients with COPD. ${ }^{4}$ However, which interventions should be included in an integrated disease management program remains unclear.

With the purpose of decreasing readmissions and improving the care of patients after COPD exacerbations, we established a multicomponent post-discharge integrated disease management program at the Cleveland Clinic. The intervention consisted of 2 parts: a multidisciplinary outpatient follow-up in the exacerbation clinic within 5-7 d of discharge and a care coordination program. This study presents a retrospective analysis of the 30- and 90-d readmission rates after initiation of the post-discharge integrated disease management program and of the specific components of the program that were associated with reduced rates of readmission.

\section{Methods}

The Cleveland Clinic Main Campus Hospital is a tertiary care academic medical center with 1,400 beds. In April 2014, a COPD exacerbation clinic was established to provide multidisciplinary out-patient follow-up for patients within 1 week of discharge from the Main Campus Hospital. A study protocol was approved by the Cleveland Clinic institutional review board (approval 15-454), and informed consent was waived. During the 1-h exacerbation clinic visit, the subject was seen by a mid-level provider, a physician, and an educator. The evaluation is standardized and includes a detailed history, medication reconciliation, review of systems, physical examination, spirometry, and 6-min walk test. Subjects also receive education on inhaler technique utilizing the teach-back method, smoking cessation counseling, pulmonary rehabilitation referral, vaccinations, and referral to relevant specialty clinics when significant comorbidities are identified that require additional attention.

In July 2014, the program was expanded to include the closer attention of a care coordinator. The care coordinator, a registered respiratory therapist, visited each subject during the hospitalization and, using a structured interview to assess health status and needs, made weekly telephone calls after discharge for up to $30 \mathrm{~d}$. When worsening respiratory status or other clinical needs were identified from these telephone interviews, the subject's primary physician was notified for intervention. Subjects were also given a COPD care coordination card, which included the coordinator's contact information.

The asynchronous introduction of different elements of the post-discharge integrated disease management follow-up program and subjects' preferences for participation

\section{QUICK LOOK}

\section{Current knowledge}

COPD readmissions represent a significant burden to health-care expenditure and patient morbidity. Integrated disease management systems have been shown to improve quality of life and reduce hospital readmission rates. Given the heterogeneity of interventions reported in previous literature, it remains unclear which specific interventions have been the most beneficial.

\section{What this paper contributes to our knowledge}

The establishment of a multicomponent post-discharge integrated disease management program consisting of a follow-up clinic appointment with a pulmonologist and/or care coordination was associated with a reduction in 90-d readmissions. Our data demonstrate that enrolling in any component of the program was beneficial. Prior health-care utilization and lung function impairment were found to be predictors for hospital readmission.

permitted an analysis of the impact of the program's individual components (ie, the exacerbation clinic and the care coordination). In this context, 4 groups were evaluated: subjects who attended the exacerbation clinic only, subjects who received the care coordination only, subjects who received both components, and subjects who received neither despite being referred.

Candidate variables for association with readmission were selected based on review of previous literature related to COPD exacerbation ${ }^{5-8}$ and investigator judgment. Data were collected regarding demographic variables, socioeconomic status, prior health-care utilization, pulmonary function test results, comorbidities, smoking status, domiciliary oxygen use, and prior history use of noninvasive ventilation in the acute setting.

\section{Statistical Analysis}

The study variables were described using sample means with SD or proportions as appropriate. The study group was divided into 2 groups based upon the outcome of 30 or 90-d readmission. Categorical variables were compared using the Pearson chi-square test or Fisher exact test, whereas continuous variables were compared using the 2 -sample independent $t$ test. To build a multivariate logistic regression model, univariate logistic regression was first performed for each of the variables. ${ }^{9}$ A complete list of the variables for univariate logistic regression analysis is provided in e-Tables 1 and 2 (see the supplementary 
materials at http://www.rcjournal.com). The predictive variables significant at $P<.10$ on univariate analysis were identified as potential predictor variables and then entered into a multivariate model. Correlation analysis was conducted to avoid multi-collinearity. A stepwise variable selection procedure was performed to select a significant subset of predictors. Two multivariate models were created for 30- and 90-d readmissions separately, and regression equations were constructed with the significant predictors, respectively. Pairwise comparisons were conducted to detect any differences among the different components of the program based on the final models. Receiver operating characteristic curves were constructed to compare the predictive capabilities of 30- and 90-d readmissions. Kaplan-Meier survival analysis and Cox proportional hazard regression analysis were also performed for the time from the clinic visit to $30-$ and $90-\mathrm{d}$ readmission, respectively. The model-building strategy for the multivariate Cox regression was the same as that for the multivariate logistic regression. The proportional hazard assumption was evaluated, and regression equations were constructed with the significant predictors. All analyses were performed by using SAS 9.4 for Linux (SAS, Cary, North Carolina). Values of $P<.05$ (2-tailed) were considered statistically significant.

\section{Results}

A total of 185 ambulatory patients were referred to the program over a 1-y period. Twenty-one patients were excluded from the analysis because they were readmitted before their exacerbation clinic appointment and therefore did not have an opportunity to complete the clinic visit. An additional 4 patients were excluded because they opted to receive local medical care and did not return to the Cleveland Clinic system. In total, 160 subjects completed 90-d follow-up and were included in the analysis.

Baseline characteristics for all groups combined are displayed in Table 1 . The mean age was $65.9 \pm 10.0 \mathrm{y}$ with mean post-bronchodilator $\mathrm{FEV}_{1}$ percent of predicted of $50 \pm 20 \% ; 52.5 \%$ were female, and $71.9 \%$ were African American. Within the year before the index admission, study subjects had been hospitalized on average $2.8 \pm 3.0$ times, attended $1.7 \pm 2.4$ pulmonary out-patient visits, and had $3.0 \pm 3.1$ primary care physician visits.

Specific clinical interventions performed during the exacerbation clinic visit included: changes to pharmacotherapy $(38 \%)$, administration of vaccines $(15 \%)$, smoking cessation counseling (10\%), oxygen prescription initiation or adjustment (4\%), instruction regarding tracheobronchial clearance techniques $(4 \%)$, pulmonary hypertension assessment $(2.5 \%)$, and provision of home bi-level positive airway pressure instructions $(2.5 \%)$. Referrals to various subspecialties were made, including to psychiatry (8\%)
Table 1. Subject Baseline Characteristics for All Groups Combined

\begin{tabular}{|c|c|}
\hline Characteristics & Values \\
\hline Age, mean \pm SD y & $65.9 \pm 10.0$ \\
\hline \multicolumn{2}{|l|}{ Sex, $n(\%)$} \\
\hline Male & $76(47.5)$ \\
\hline Female & $84(52.5)$ \\
\hline \multicolumn{2}{|l|}{ Race, $n(\%)$} \\
\hline African American & 115 (71.9) \\
\hline Caucasian & $43(26.9)$ \\
\hline Other & $2(1.3)$ \\
\hline Income, mean $\pm \mathrm{SD} \$ \mathrm{US}$ & $30,973 \pm 18,549$ \\
\hline $\mathrm{BMI}$, mean $\pm \mathrm{SD} \mathrm{kg} / \mathrm{m}^{2}$ & $28.0 \pm 9.0$ \\
\hline Current smoker, $n(\%)$ & $52(32.5)$ \\
\hline \multicolumn{2}{|l|}{ Prednisone use, $n(\%)$} \\
\hline Chronic & $25(15.6)$ \\
\hline Intermittent & $110(68.8)$ \\
\hline None & $25(15.6)$ \\
\hline Supplemental oxygen use, $n(\%)$ & $101(63.1)$ \\
\hline NIV use, $n(\%)$ & $49(30.6)$ \\
\hline $\begin{array}{l}\text { Post-bronchodilator } \mathrm{FEV}_{1}, \\
\text { mean } \pm \mathrm{SD} \mathrm{L}\end{array}$ & $1.1 \pm 0.6$ \\
\hline $\begin{array}{l}\text { Post-bronchodilator } \mathrm{FEV}_{1}, \\
\text { mean } \pm \mathrm{SD} \% \text { predicted }\end{array}$ & $50 \pm 20$ \\
\hline $\begin{array}{l}\text { Post-bronchodilator FVC, } \\
\text { mean } \pm \text { SD \% predicted }\end{array}$ & $70 \pm 20$ \\
\hline $\mathrm{D}_{\mathrm{LCO}}$, mean $\pm \mathrm{SD} \%$ predicted & $40 \pm 20$ \\
\hline $\begin{array}{l}\text { No. of hospitalizations in previous } \\
\text { year, mean } \pm \text { SD }\end{array}$ & $2.8 \pm 3.0$ \\
\hline $\begin{array}{l}\text { No. of pulmonary clinic visits in } \\
\text { previous year, mean } \pm \text { SD }\end{array}$ & $1.7 \pm 2.4$ \\
\hline $\begin{array}{l}\text { No. of PCP clinic visits in previous } \\
\text { year, mean } \pm \text { SD }\end{array}$ & $3.0 \pm 3.1$ \\
\hline Cancer, $n(\%)$ & $29(18.1)$ \\
\hline Anxiety, $n(\%)$ & $27(16.9)$ \\
\hline Cirrhosis, $n(\%)$ & $2(1.3)$ \\
\hline Atrial fibrillation/atrial flutter, $n(\%)$ & $34(21.3)$ \\
\hline Diabetes mellitus, $n(\%)$ & $58(36.3)$ \\
\hline Diabetes with neuropathy, $n(\%)$ & $15(9.4)$ \\
\hline Pulmonary fibrosis, $n(\%)$ & $2(1.3)$ \\
\hline Coronary artery disease, $n(\%)$ & $45(28.1)$ \\
\hline Congestive heart failure, $n(\%)$ & $48(30.0)$ \\
\hline Gastroesophageal reflux disease, $n(\%)$ & $45(28.1)$ \\
\hline Peptic ulcer disease, $n(\%)$ & $11(6.9)$ \\
\hline Hypertension, $n(\%)$ & $107(66.9)$ \\
\hline Hyperlipidemia, $n(\%)$ & $73(45.6)$ \\
\hline Chronic renal disease, $n(\%)$ & $31(19.4)$ \\
\hline Cor pulmonale, $n(\%)$ & $22(13.8)$ \\
\hline Asthma, $n(\%)$ & $38(23.8)$ \\
\hline Obstructive sleep apnea, $n(\%)$ & $42(26.3)$ \\
\hline $\begin{array}{l}N=160 . \\
\text { BMI = body mass index } \\
\text { NIV = noninvasive ventilation } \\
\text { D }_{\text {LCO }=\text { diffusing capacity of the lung for carbon mor }} \\
\text { PCP = primary care physician }\end{array}$ & \\
\hline
\end{tabular}




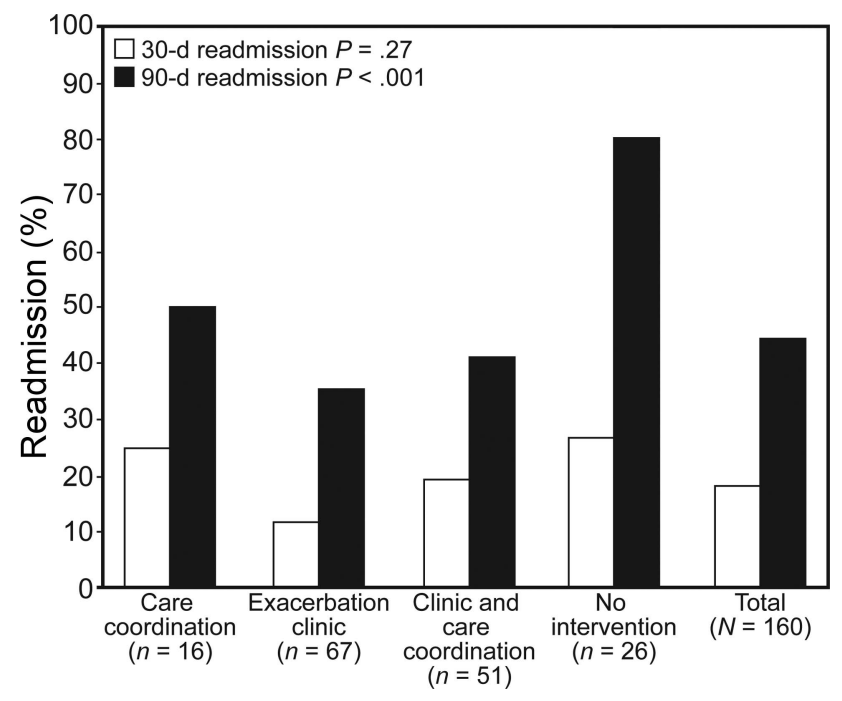

Fig. 1. Thirty- and 90-d readmission rates for different components of the COPD care coordination program. $P$ values use the Fisher exact test, comparing any intervention with no intervention.

and sleep medicine (8\%). Also, $89 \%$ of subjects received referrals for pulmonary rehabilitation.

Thirty- and 90-d readmission rates for the 4 groups are displayed in Figure 1. For all 160 subjects, the total 30and $90-\mathrm{d}$ readmission rates were 18.1 and $46.2 \%$, respectively. In the individual intervention groups, the 30- and 90-d readmission rates were: exacerbation clinic $(n=67)$, 11.9 and $35.8 \%$; care coordination $(n=16), 25.0$ and $50.0 \%$; both $(n=51), 19.6$ and $41.2 \%$; neither $(n=26)$, 26.9 and $80.8 \%$, respectively.

Univariate logistic regression analysis for 30- and 90-d readmission identified 12 and 16 variables as significant predictors of readmission, respectively (e-Tables 3 and 4). These variables were then used to create a multivariate model.

The final multivariate logistic regression model identified 2 predictor variables of 30-d readmission: number of prior hospitalizations (odds ratio [OR] 1.36 [95\% CI 1.171.57]) and use of noninvasive ventilation (OR 3.07 [95\% CI 1.19-7.91]). As shown in Figure 2, the model's C statistic was 0.84 , suggesting excellent predictive accuracy (C statistic values of $0.8-0.9$ indicate excellent discrimination ${ }^{9}$ ). The final multivariate logistic regression model for 90-d readmission identified 3 predictor variables: receiving any component of the post-discharge integrated disease management program (care coordination vs neither: OR $0.08,95 \%$ CI $0.01-0.51$; exacerbation clinic vs neither: OR $0.09,95 \%$ CI $0.02-0.33$; both versus neither: OR $0.11,95 \%$ CI $0.03-0.43$, number of primary care physician visits within the previous year OR $1.25,95 \% \mathrm{CI}$ $1.09-1.45$, and number of hospitalizations within the previous year OR $1.90,95 \%$ CI $1.48-2.43$ with a C statistic of 0.87 (Fig. 3). Pairwise comparison did not demonstrate

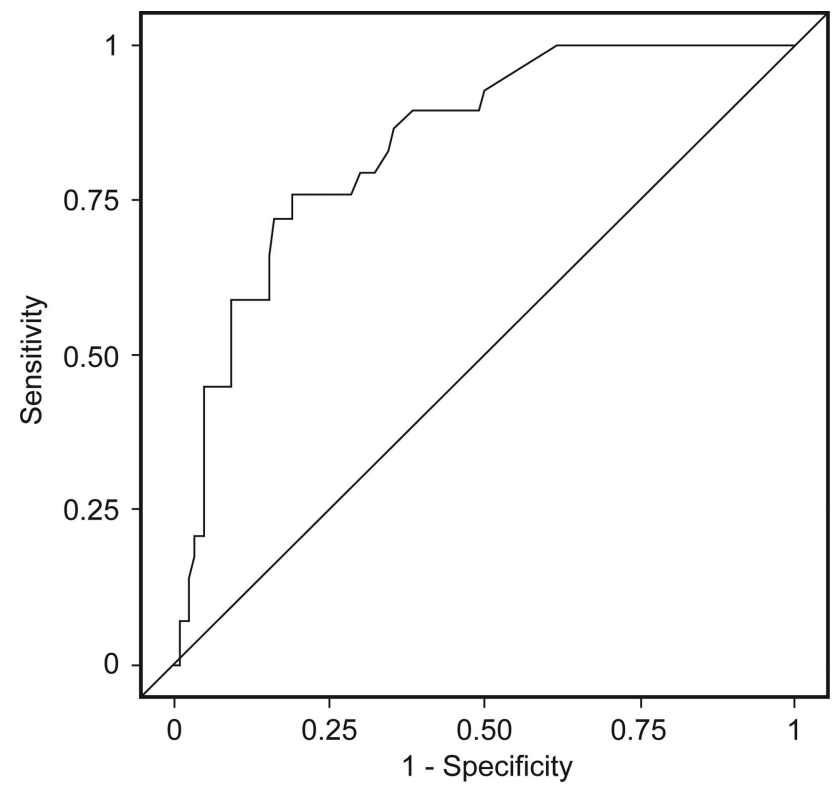

Fig. 2. Receiver operating characteristic curve for 30-d readmission. C statistic $=0.84$, and area under the curve $=0.843$.

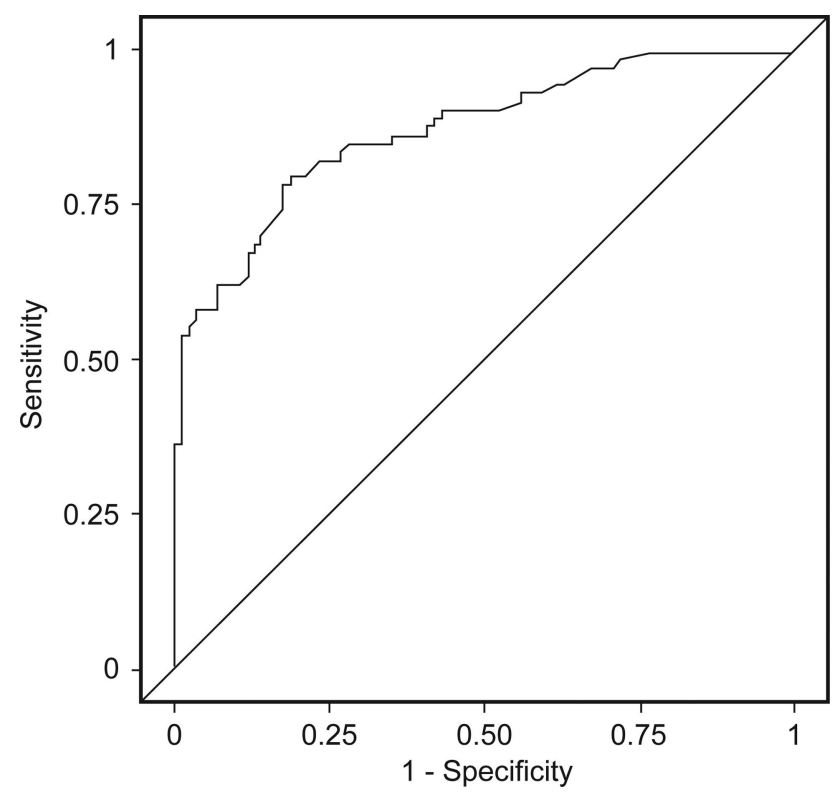

Fig. 3. Receiver operating characteristic curve for 90-d readmission. C statistic $=0.87$, and area under the curve $=0.873$.

a significant difference between the individual program components (Table 2).

Cox proportional hazard analysis identified 4 predictor variables of 30-d readmission: percent of predicted postbronchodilator $\mathrm{FEV}_{1}$ with hazard ratio (HR) $0.09,95 \% \mathrm{CI}$ 0.01-0.69, number of hospitalizations within the previous year (HR 1.27, 95\% CI 1.17-1.38), diabetes mellitus (HR 2.28, 95\% CI 1.12-4.65), and peptic ulcer disease (HR $3.15,95 \%$ CI $1.15-8.63)$. Four predictors were identified 
Table 2. Pairwise Comparison of Each Component of the Intervention Group

\begin{tabular}{lc}
\hline \hline \multicolumn{1}{c}{ Component } & $P$ \\
\hline Care coordination vs no intervention & .007 \\
Exacerbation clinic vs no intervention & $<.001$ \\
Clinic and care coordination vs no intervention & .002 \\
Care coordination vs clinic and care coordination & .70 \\
Clinic and care coordination vs exacerbation clinic & .64 \\
Care coordination vs exacerbation clinic & .93 \\
\hline
\end{tabular}

for 90-d readmission: receiving any component of the post-discharge integrated disease management program (care coordination: HR $0.39,95 \%$ CI $0.17-0.89$; exacerbation clinic: HR $0.25,95 \%$ CI $0.14-0.44$; both: HR $0.33,95 \%$ CI 0.17-0.67), number of hospitalizations in the previous year (HR 1.20, 95\% CI 1.13-1.27), number of primary care physician visits within the previous year (HR 1.12, 95\% CI 1.02-1.22), and hypertension (HR 2.15, 95\% CI 1.24-3.73). After adjusting for explanatory variables, the probability of being readmitted within $90 \mathrm{~d}$ was significantly lower when receiving any program component compared with receiving no intervention $(P<.05)$ (Fig. 4).

\section{Discussion}

In this retrospective analysis of a post-discharge integrated disease management program, enrolling in any component of the program was associated with reduced 90-d readmission. Our data are consistent with previous reports that demonstrate that integrated care confers greater benefit for mid-term than for short-term outcomes. ${ }^{10}$

Our study extends current knowledge about integrated care management by suggesting specific components that confer benefit in averting readmission for COPD. Although a meta-analysis that included 26 randomized, controlled trials involving 2,997 subjects concluded that post-discharge integrated disease management programs resulted in an improvement in quality of life and functional status and a reduction in the number of hospital admissions over a 3-12-month period when compared with controls, ${ }^{4}$ the significant heterogeneity of integrated management interventions throughout the various studies precluded ascertaining which specific interventions were effective. In our study, we found that receiving any component of the postdischarge integrated disease management program was associated with lower $90-\mathrm{d}$ readmission than receiving no intervention, with no difference among those receiving care coordination alone, attending the exacerbation clinic alone, or receiving both components (Fig. 4). Our findings agree with prior observations that early pulmonary follow-up after COPD exacerbations can avert readmissions. For instance, in a retrospective cohort study, Gavish et al ${ }^{11}$ reported that not attending the follow-up visit with a pulmonologist within 1 month of discharge was associated with an increased risk of 90-d readmission. The benefit of early post-discharge follow-up is also supported for other disease states. Hernandez et al ${ }^{12}$ reported a lower risk of 30-d readmission for subjects with heart failure when follow-up occurred within $7 \mathrm{~d}$ of discharge. Similar results were also seen with early follow-up after high risk surgery ${ }^{13}$ and with sickle cell disease. ${ }^{14}$ Unfortunately, not all patients can attend an early follow-up appointment despite the demonstrated benefits. In this context, Gavish et al ${ }^{11}$ identified distant residence as a risk factor for 90-d readmission. In our study, subjects who received only care coordination without a clinic visit had lower 90-d readmission rates, suggesting that patients who are unable to make an out-patient follow-up visit still experience benefit, perhaps because the care coordinator helped to craft an individualized post-discharge clinical plan.

In contrast to the observations regarding 90 -d readmission, no reduction in 30-d readmission rates was observed for subjects enrolled in the post-discharge integrated disease management program. Although our data do not permit a firm conclusion, we speculate that a shorter-term benefit may not have been observed because the impact of the intervention is cumulative, and a difference may not have been detectable until the second and third months post-discharge. For instance, some interventions that have been shown to reduce hospital admissions, such as pulmonary rehabilitation, ${ }^{15}$ may not be available to patients within the first $30 \mathrm{~d}$ after discharge. In our program, although a pulmonary rehabilitation order was placed for $89 \%$ of the subjects, $<10 \%$ started the program within $30 \mathrm{~d}$. Even if these patients were recruited within $30 \mathrm{~d}$, it would have been unlikely for them to accrue the expected benefits within this short time frame. The general barriers to enrollment for others included access to transportation, patient refusal, work commitment, and having undergone pulmonary rehabilitation before (ie, no available reimbursement). In keeping with this low participation, Jones et al ${ }^{16}$ reported that only $32 \%$ of eligible subjects were referred to pulmonary rehabilitation after COPD exacerbations, and only $9.6 \%$ actually completed the program. Improving access and adherence to pulmonary rehabilitation programs in the real-world practice setting may lead to overall reduced health-care utilization.

We found a strong association between the likelihood of 30- and 90-d readmission and previous health-care utilization. In keeping with prior observations, lung function impairment and past health-care utilization were strongly associated with 30- and 90-d readmissions. For example, in a study of 172 subjects admitted for acute COPD, the number of COPD admissions in the prior year was associated with a $42 \%$ increase in risk of $30-\mathrm{d}$ readmission for each additional past hospitalization. ${ }^{17}$ Similarly, Almagro 


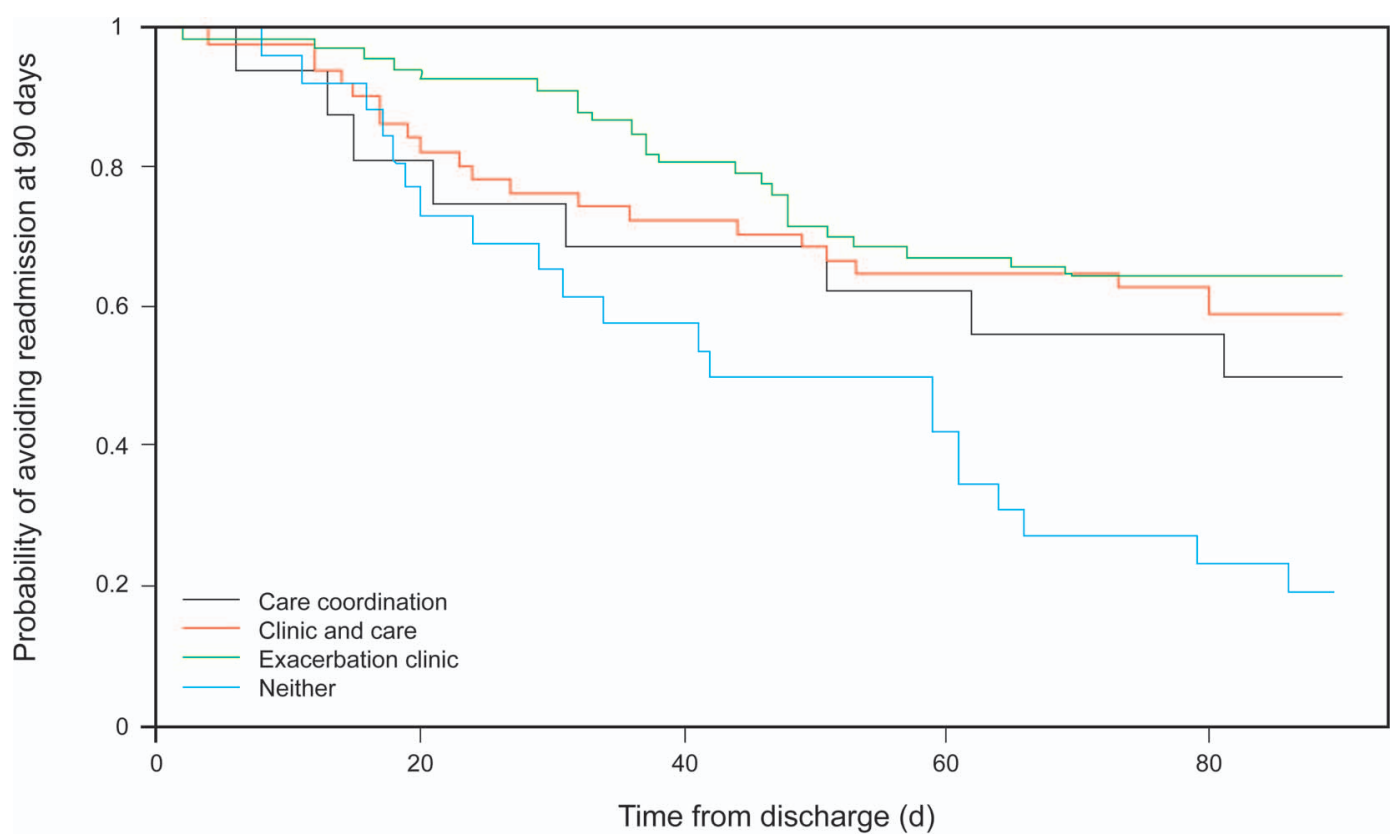

Fig. 4. Kaplan-Meier survival curve for the different components of the COPD care coordination program for $90-\mathrm{d}$ readmission. $P<.001$.

et $\mathrm{al}^{8}$ demonstrated that the best predictor for COPD readmission was the number of hospitalizations within the previous year (OR 4.27, 95\% CI 1.5-12).

In keeping with previous observations, lung function impairment was associated with $30-\mathrm{d}$ readmission in our study. In the ECLIPSE study, ${ }^{18}$ which assessed 2,138 COPD subjects, exacerbations of COPD were independently associated with worse lung function. Although the ECLIPSE study also identified gastroesophageal reflux disease as a predictor for future exacerbations of COPD, such an association with 30- and 90-d readmissions was not observed in our cohort. The incidence of gastroesophageal reflux disease (as ascertained from the medical record) was $27 \%$ in our population (comparable with ECLIPSE participants), and $78 \%$ of subjects were on anti-reflux therapy. Since treatment of gastroesophageal reflux disease has been associated with reduced exacerbation frequency, ${ }^{19}$ it is plausible that any conferred risk for exacerbation was abrogated with the high rate of gastroesophageal reflux disease therapy in our cohort.

During the clinic visit, a change to pharmacotherapy compliant with Global Initiative for Chronic Obstructive Lung Disease (GOLD) guidelines ${ }^{20}$ was made $38 \%$ of the time. We speculate that the apparently high frequency with which discharged subjects' regimens were changed may reflect an unawareness of treatment guidelines by the discharging physicians. A retrospective analysis of United States COPD and Medicare patients demonstrated that over two thirds of patients were not prescribed maintenance COPD pharmacotherapy per GOLD guidelines during a 1-y study. ${ }^{21}$ Furthermore, a survey of primary care physicians by Foster et $\mathrm{a}^{22}$ showed that only $55 \%$ were aware of COPD guidelines and only $25 \%$ would use such guidelines for decision making. Clearly, a benefit of follow-up clinics is the opportunity to optimize patients' drug regimens.

Several limitations of our study warrant comment. First, by virtue of its retrospective nature, our data are hypothesis-generating and require validation in a prospective, pragmatic clinic trial. Second, because the study was conducted in a single center, albeit large, the generalizability of our findings requires validation in other settings. As a matter of internal bias, ${ }^{23}$ inclusion of only those subjects referred to the post-discharge integrated disease management raises the specter of selection bias in our sample. On the other hand, the readmission rates for the entire group were similar to those reported in the literature, suggesting that the groups were comparable in terms of susceptibility to the outcomes of interest. ${ }^{1}$ It could also be argued that subjects who participated in the post-discharge integrated disease management program were more likely to be adherent to medical care than those who did not, hence biasing the results toward a favorable impact. Although we have accounted for some of the factors that have traditionally been related to adherence (eg, the presence of multiple comorbidities and socioeconomic status) in our multivariate analysis, the possible impact of subject nonadherence on the outcome is not entirely excluded.

\section{Conclusions}

To our knowledge, the establishment of a multidisciplinary COPD exacerbation clinic after discharge under 
the direction of a pulmonologist is a novel intervention. Utilizing 2 different analytic approaches (absolute event rate and survival models), short-term improvement (ie, lower 30-d readmission rates) was not observed, whereas longer-term benefits were evident. In this regard, our findings suggest that the focus of Centers for Medicare and Medicaid Services reimbursement withholding might shift from a 30-d readmission to a 90-d readmission focus, where intervention can effect improvement. Overall, our findings suggest that the interventions of a post-discharge COPD follow-up clinic visit and care coordination by a respiratory therapist are feasible and, subject to further validation, effective.

\section{REFERENCES}

1. Ford ES. Hospital discharges, readmissions, and ED visits for COPD or bronchiectasis among US adults: findings from the nationwide inpatient sample 2001-2012 and nationwide emergency department sample 2006-2011. Chest 2015;147(4):989-998.

2. Dalal AA, Christensen L, Liu F, Riedel AA. Direct costs of chronic obstructive pulmonary disease among managed care patients. Int J Chron Obstruct Pulmon Dis 2010;5:341-349.

3. Shah T, Churpek MM, Coca Perraillon M, Konetzka RT. Understanding why patients with COPD get readmitted: a large national study to delineate the Medicare population for the readmissions penalty expansion. Chest 2015;147(5):1219-1226.

4. Kruis AL, Smidt N, Assendelft WJJ, et al. Integrated disease management interventions for patients with chronic obstructive pulmonary disease. Cochrane Database Syst Rev 2013;(10):CD009437.

5. Divo M, Cote C, de Torres JP, Casanova C, Marin JM, Pinto-Plata $\mathrm{V}$, et al. Comorbidities and risk of mortality in patients with chronic obstructive pulmonary disease. Am J Respir Crit Care Med 2012; 186(2):155-161

6. Bahadori K, FitzGerald JM, Levy RD, Fera T, Swiston J. Risk factors and outcomes associated with chronic obstructive pulmonary disease exacerbations requiring hospitalization. Can Respir J 2009; 16(4):e43-e49.

7. Celli BR, Cote CG, Marin JM, Casanova C, Montes de Oca M, Mendez RA, et al. The body mass index, airflow obstruction, dyspnea and exercise capacity index in chronic obstructive pulmonary disease. N Engl J Med 2004;350(10):1005-1012.

8. Almagro P, Barreiro B, Ochoa de Echaguen A, Quintana S, Rodríguez Carballeira M, Heredia JL, Garau J. Risk factors for hospital readmission in patients with chronic obstructive pulmonary disease. Respiration 2006;73(3):311-317.
9. Hosmer DW, Lemeshow S. Applied logistic regression, 2nd Ed. New York: John Wiley \& Sons; 2000.

10. Shorofsky M, Lebel M, Sedeno M, Zhi Li P, Bourbeau J. Discharge care bundle for patients with acute exacerbation of COPD: benefits more likely to be seen beyond 30 days. Int J Respir Pulm Med. 2015;2(3):2-6.

11. Gavish R, Levy A, Dekel OK, Karp E, Maimon N. The association between hospital readmission and pulmonologist follow-up visits in patients with COPD. Chest 2015;148(2):375-381.

12. Hernandez AF, Greiner MA, Fonarow GC, Hammill BG, Heidenreich PA, Yancy $\mathrm{CW}$, et al. Relationship between early physician follow-up and 30-day readmission among Medicare beneficiaries hospitalized for heart failure. JAMA 2010;303(17):1716-1722.

13. Brooke BS, Stone DH, Cronenwett JL, Nolan B, DeMartino RR, MacKenzie TA, et al. Early primary care provider follow-up and readmission after high-risk surgery. JAMA Surg 2014;149(8):821828.

14. Leschke J, Panepinto JA, Nimmer M, Hoffmann RG, Yan K, Brousseau DC. Outpatient follow-up and rehospitalizations for sickle cell disease patients. Pediatr Blood Cancer 2012;58(3):406-409.

15. Puhan MA, Gimeno-Santos E, Scharplatz M, et al. Pulmonary rehabilitation following exacerbations of chronic obstructive pulmonary disease. Cochrane Database Syst Rev. 2009;(1):CD005305.

16. Jones SE, Green SA, Clark AL, Dickson MJ, Nolan AM, Moloney $\mathrm{C}$, et al. Pulmonary rehabilitation following hospitalisation for acute exacerbation of COPD: referrals, uptake and adherence. Thorax 2014; 69(2):181-182

17. Jennings JH, Thavarajah K, Mendez MP, Eichenhorn M, Kvale P, Yessayan L. Predischarge bundle for patients with acute exacerbations of COPD to reduce readmissions and ED visits: a randomized controlled trial. Chest 2015;147(5):1227-1234.

18. Hurst JR, Vestbo J, Anzueto A, Locantore N, Müllerova H, TalSinger R, et al. Susceptibility to exacerbation in chronic obstructive pulmonary disease. N Engl J Med 2010;363(12):1128-1138.

19. Sasaki T, Nakayama K, Yasuda H, Yoshida M, Asamura T, Ohrui T, et al. A randomized, single-blind study of lansoprazole for the prevention of exacerbations of chronic obstructive pulmonary disease in older patients. J Am Geriatr Soc 2009;57(8):1453-1457.

20. Vestbo J, Hurd SS, Agustí AG, Jones PW, Vogelmeier C, Anzueto A, et al. Global strategy for the diagnosis, management, and prevention of chronic obstructive pulmonary disease: GOLD executive summary. Am J Respir Crit Care Med 2013;187(4):347-365.

21. Make B, Dutro MP, Paulose-Ram R, Marton JP, Mapel DW. Undertreatment of COPD: a retrospective analysis of US managed care and Medicare patients. Int J Chron Obstruct Pulmon Dis 2012;7:1-9.

22. Foster JA, Yawn BP, Maziar A, Jenkins T, Rennard SI, Casebeer L. Enhancing COPD management in primary care settings. MedGenMed 2007;9(3):24.

23. Feinstein A. The architecture of clinical research, Vol 14. New York: Wiley Subscription Services; 1985:50.

This article is approved for Continuing Respiratory Care Education credit. For information and to obtain your CRCE

(free to AARC members) visit www.rcjournal.com

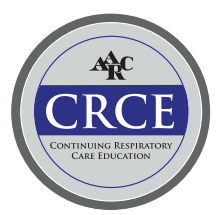

